\title{
Regulación ambiental sobre los productos farmacéuticos residuales en ambientes acuáticos
}

\section{Environmental regulation on residual pharmaceutical products in aquatic environments}

\author{
Diego Ivan Caviedes Rubio \\ Magister en Ecología y Gestión de Ecosistemas Estratégicos \\ Universidad Cooperativa de Colombia \\ diego.caviedesr@campusucc.edu.co \\ Daniel Ricardo Delgado \\ PhD en Ciencias Farmacéuticas \\ Universidad Cooperativa de Colombia \\ danielr.delgado@campusucc.edu.co
}

\section{Introducción}

Los productos farmacéuticos activos representan un grupo de contaminantes, que aunque en pequeñas cantidades en el medio ambiente, son motivo de preocupación ya que están diseñados para ser intrínseca y biológicamente activos y potencialmente estables en virtud de los procesos metabólicos. Hoy en día, se reconoce por la comunidad científica que la exposición continua de dosis bajas de productos farmacéuticos puede producir efectos a largo plazo sobre el medio ambiente como consecuencia de posibles daños irreversibles del ecosistema y la salud humana (Silva et al; 2012. Rahman et al; 2009a). Debido a la alta polaridad y baja volatilidad, la mayoría de los productos farmacéuticos tienden a ser fácilmente transportados y descargados en los cuerpos de agua (Zhang et al, 2014).

La presencia de los productos farmacéuticos en el medio ambiente se observó por primera vez en la década de 1980, algunos residuos farmacéuticos se detectaron en afluentes y efluentes de plantas de tratamiento de aguas residuales domésticas y en aguas superficiales (Lacina et al, 2013). De igual manera, se han detectado concentraciones medibles de hormonas y antibióticos en el suelo y las aguas subterráneas y superficiales que reciben el escurrimiento de los campos fertilizados con estiércol y aguas abajo de las operaciones con animales de granja, generados por el uso excesivo de antibióticos en la industria ganadera y el cambio espectacular en los últimos años a concentrados alimenticios para animales, lo que conlleva al aumento concomitante en el volumen de los desechos animales por unidad de superficie (Lee et al, 2009; Cai et al, 2012). Estos residuos se movilizan en suelos, por infiltración y escorrentía y en las aguas subterráneas, circunstancias en las que no se sabe si ellos reaccionan entre sí, así como con los pesticidas y herbicidas formando otros compuestos que, ya sea individualmente o en combinación, podrían adversamente afectar a bacterias, hongos y organismos superiores (Fisher, 2008. Kiran et al, 2011).

Evaluaciones recientes indican que hasta la fecha más de 30 productos farmacéuticos y compuestos orgánicos emergentes de preocupación se han detectado en aguas potables de todo el mundo (AlOdaini et al, 2010; Kleywegt et al, 2011. Jelic et al, 2012). La detección de estos compuestos en el agua potable se ha atribuido a su presencia en la fuente de agua abastecedora y a la incapacidad del proceso de tratamiento en el sistema de agua potable para reducirlos, lo que los convierte actualmente, en cuanto a los microcontaminantes, en el centro de atención de la salud pública, por sus efectos adversos (Kleywegt et al, 2011; Silva et al, 2012, Liu et al, 2012).

\section{Regulación Internacional}

Las actuales políticas de la Unión Europea relacionadas con la reducción del riesgo de la presencia de productos farmacéuticos en el medio ambiente, puede ser visto en el contexto de los principios de precaución y prevención, en lugar de encaminarse a la solución del problema. La Unión Europea mediante la Directiva Marco del Agua, por su sigla en inglés (WFD), ha desarrollado una lista de sustancias peligrosas prioritarias publicadas en la (Decisión 2455/2001/EC) (Unión Europea, 2001) que luego se convirtió en el anexo $\mathrm{X}$ de la directiva, con la presencia de 33 sustancias en las que no se incluyó productos farmacéuticos, por lo que estas sustancias no se encuentran dentro de un programa de vigilancia. Por su parte, La Directiva sobre agua potable (DWD) enumera 48 parámetros de calidad para agua de consumo humano (Kampa et al 2010), en los que no se 
incluye ningún fármaco, sin embargo, los Estados miembros son libres de establecer valores para nuevos parámetros no incluidos en la lista de la DWD, en cuando sea necesario para la protección de la salud humana.

Los avances en el campo regulatorio se han limitado a exigir mediante la Directiva (2004/27/CE) (Unión Europea, 2004) una evaluación de impacto ambiental para cada medicamento de consumo humano que se comercialice y en el caso de productos farmacéuticos de uso veterinario un análisis de riesgo-beneficio para la seguridad del consumidor y el ambiente, de igual manera, esta directiva establece que todos los Estados miembros de la Unión Europea, deben establecer sistemas de recolección para recuperación y la disposición segura de los medicamentos no utilizados y caducados. En septiembre de 2010, el Parlamento Europeo aprobó enmiendas a la legislación sobre farmacovigilancia (Directiva 2001/83/CE y el Reglamento CE No 726/2004) a fin de ampliar su concepto a las preocupaciones ambientales y para considerar medidas para controlar y evaluar el riesgo de los efectos ambientales de los medicamentos (Silva et al, 2012. Verlicchi et al, 2012).

Actualmente la WFD exige tres programas de monitoreo en las masas de aguas superficiales de la Unión Europea, estos se refieren inicialmente a un control de vigilancia, cuyo monitoreo se realiza generalmente cada 6 años, con el objetivo de complementar y validar un análisis de impacto, apoyar futuros programas de control y evaluación de las condiciones ambientales cambiantes generadas por la actividad antropogénica (Quevauviller \& Carere, 2012). Con el objetivo de establecer el estado de las masas de agua en riesgo de no cumplir los objetivos ambientales de la WFD se exige el control operativo, cabe aclarar que este se basa en los estándares de calidad ambiental para 53 contaminantes químicos seleccionados como sustancias prioritarias de preocupación en toda la Unión Europea (Unión Europea, 2013), en los que no se registran fármacos, y finalmente se realiza el control de investigación para determinar la magnitud y el impacto de contaminaciones accidentales; las herramientas de monitoreo empleadas para estos controles corresponden básicamente a bioensayos, biomarcadores y métodos ecológicos, para determinar toxicidad, respuesta biológica a nivel celular o individual y o cambios a nivel poblacional o en las comunidades, respectivamente (Wernersson et al, 2015).

Uno de los escenarios más autorizadas para las discusiones sobre los contaminantes emergentes en la actualidad es el Norman Network, iniciativa que nace de un proyecto de colaboración a gran escala a través de muchos institutos de investigación europea (Norman, 2012). La red Norman ha elaborado una lista de más de 700 sustancias individuales divididas en 25 clases basadas en frecuencia de detección en muestras ambientales. Esta es actualmente la lista más completa de los contaminantes jamás compilada. Dentro de
Norman, los investigadores se han comprometido a desarrollar y supervisar las técnicas analíticas, pruebas de toxicidad, y la evaluación de los impactos en el ecosistema. Como se detalla en el documento de 2013 del Programa de Trabajo-Medio Ambiente bajo el séptimo marco esquema de financiación de los programas de la Unión Europea, un gran enfoque está dado en Europa en el desarrollo de la vigilancia y la tecnología de reducción de emisiones, especialmente centrándose en varias clases de sustancias de emergente preocupación ambiental como sustancias persistentes, bioacumulativas y toxicas (PBT) (Wennmalm \& Gunnarsson, 2010), en particular, estos incluyen productos farmacéuticos y de higiene personal (PPCPs) emitidos por las aguas residuales municipales y hospitales, que representan las más numerosas clases dentro de la Lista de Norman (Sharma et al, 2014a). Estas iniciativas generaran el soporte científico que requieren los procesos de construcción de la normatividad específica a establecer en países en desarrollo e incluso algunos desarrollados, como India, China, Brasil, Sudáfrica entre otros, quienes realizan esfuerzos escasamente por cumplir con lo convenido en los tratados de Estocolmo sobre contaminantes orgánicos persistentes, con un total de 179 partes ratificadas (COP)(FAO \& PNUMA, 2015. Manickum \& John, 2014. PNUMA, 2013) y Basilea con 181 partes o países vinculados, el cuales reglamenta los movimientos transfronterizos de desechos peligrosos y su eliminación (Sharma et al, 2014b. PNUMA, 2014).

En los Estados Unidos de Norte América, la regulación de los productos farmacéuticos en el medio ambiente está dentro del ámbito tanto de la Agencia de Protección Ambiental de EE.UU. (EPA), que tiene autoridad sobre la mayoría de los ámbitos del medio ambiente a través de la Ley de Agua Limpia (CWA), Ley de Aire Limpio (CAA), Ley Control de Sustancias Tóxicas (TSCA) y Conservación y Recuperación de Recursos (RCRA), entre otros, y la FDA, que tiene autoridad sobre productos farmacéuticos a través de la Ley Federal de Alimentos, Medicamentos y Cosméticos (FFDCA) con la responsabilidad reglamentaria para investigar el impacto medioambiental de los productos farmacéuticos a través de una de las leyes ambientales más antiguas, la Nacional de Medio Ambiente Policy Act de 1969 (NEPA), que requiere de cualquier entidad gubernamental federal de los EE.UU. para evaluar el impacto de las acciones sobre el medio ambiente (McVey 2012.); esta es a su vez, supervisada por el Consejo de la Casa Blanca de Política y Calidad Ambiental y los EE.UU. EPA.

Actualmente la EPA en su gestión por la planificación de programas de directrices sobre efluentes industriales realiza estudios sobre las prácticas de eliminación de productos farmacéuticos en los hospitales, hospicios, centros de atención y clínicas u hospitales veterinarios, con el objetivo de identificar las prácticas más eficientes dirigidas a reducir los vertimientos de residuos farmacéuticos (USEPA, 2014a); de igual manera, la agencia ha propuesto una 
lista de sustancias candidatas a contaminantes (CCL4) de 100 contaminantes químicos o grupos de contaminantes químicos y 12 contaminantes microbianos, que hacen parte de productos farmacéuticos y de higiene personal entre otros (USEPA, 2014b. USEPA, 2015), los cuales se están evaluando para determinar la necesidad de regulación especial sobre el agua potable a fin de proteger la salud pública. Así mismo, la EPA ha desarrollado métodos de análisis para aproximadamente 100 productos farmacéuticos, hormonas, esteroides y otros para el cuidado personal (USEPA, 2007).

En países como Japón, Canadá y Australia, la reglamentación de la ley de protección ambiental Canadiense y Australiana y el Ministerio de Salud de Japón se enfoca en evaluaciones del riesgo ambiental que generan los productos farmacéuticos (WHO, 2012), cuyos resultados aprueban o niegan el registro y la aprobación de su comercialización y la implementación de un módulo o documento técnico que incluye una indicación de los riesgos ambientales pertinentes, hace referencia a las pruebas fisicoquímicas estándar y cualquier prueba apropiada que se lleve a cabo sobre la biodegradabilidad, incluyendo algunas pruebas en especies sensibles (Straub y Hutchinson, 2012. Marsalek, 2008), pero no incluyen un listado de productos farmacéuticos específicos a los que se vigile o regule. En China, por su parte el ministerio de protección del medio ambiente ha puesto en marcha desde el año 2013 un plan quinquenal para el control y la prevención de los riesgos ambientales de las sustancias químicas incluidos los productos farmacéuticos (Bu et al, 2013. Dai et al, 2015).

Brasil, así como los demás países latinoamericanos, presentan vacíos al momento de establecer un espectro normativo referente a la generación en la fuente, transporte, comercialización y posconsumo de productos farmacéuticos y de higiene personal (Bila, 2003. Bellan et al, 2012), que por sus características de persistencia, bioacumulacion y toxicidad son una amenaza para los ecosistemas acuáticos y para las comunidades que de allí extraen el agua de consumo. De acuerdo con (Gemiliano et al, 2006) en los países suramericanos se hace evidente la carencia en la organización de los servicios y en los dispositivos normativos o legales de la calidad de agua para consumo humano; Colombia y Brasil se encuentran dentro de los pocos de Latinoamérica y el Caribe que desarrollan acciones de vigilancia de la calidad del agua para consumo humano (INS, 2014), pero su normatividad no incluye el control de productos farmacéuticos en el agua de consumo.

\section{Regulación Colombiana.}

La legislación Colombiana, aún más que la legislación internacional, presenta vacíos respecto al control de estos residuos peligrosos ya que no incluye ninguna regulación específica sobre productos farmacéuticos en el medio ambiente acuático y solo se refiere a la prevención y gestión empresarial o institucional dentro del ciclo comercial y productivo, a ello se refiere la resolución 1164 de 2002, referente a la gestión integral de residuos hospitalarios y similares y la resolución 371 de 2009 expedida por el Ministerio de Ambiente, Vivienda y Desarrollo Territorial (MAVDT) para la implementación de los Planes de Gestión de Devolución de Productos Posconsumo de Fármacos o Medicamentos Vencidos, acciones que contribuyen a disminuir los vertimientos de estas sustancias, pero que no las regulan directamente. De igual manera no se incluye dentro de los parámetros de calidad de aguas para consumo humano establecidos en el Decreto 1575 de 2007 en el que se establece el Sistema para la Protección y Control de la Calidad del Agua para Consumo Humano y la resolución 2115 de 2007 del MAVDT, por medio de la cual se señalan características, instrumentos básicos y frecuencias del sistema de control y vigilancia para la calidad del agua para consumo humano y solo se incluyen aunque de una forma generalizada en la política para la Gestión Integral de Residuos Peligrosos (RESPEL), expedida por El Consejo Nacional Ambiental Colombiano desde el año 2005, cuyos objetivos específicos orientan su aplicación a la minimización y prevención en la generación y a promover el manejo ambientalmente adecuado de los RESPEL; en este sentido los productos farmacéuticos desechados han sido clasificados como residuos peligrosos debido a su ecotoxisidad y de acuerdo con el decreto 4741 del 30 de diciembre del 2005, por el cual se reglamenta parcialmente la prevención y el manejo de los residuos o desechos peligrosos generados en el marco de la gestión integral, el cual adopta la clasificación de estos residuos establecida en el Anexo 1 del convenio de Basilea (MAVDT, 2005).

Teniendo en cuenta que solo hasta la última década se ha observado detenidamente el riesgo ecológico y sobre la salud humana que genera la presencia de estos microcontaminantes en las fuentes de agua y que las técnicas de detección hasta ahora se están estandarizando ya que su naturaleza bioquímica es muy compleja y por tanto muy específica; razones por las cuales es difícil esperar que en Colombia exista una regulación específica para estos, aunque cabe indicar que tampoco se encuentra lejos del avance legal global que existe en este aspecto y que las políticas y demás herramientas legales en materia ambiental están adecuadas estructuralmente para la generación de una regulación específica para los microcontaminantes xenobioticos entre los que se incluyen los productos farmacológicos; siempre que se siga el principio mediante el cual la formulación de las políticas ambientales tendrán en cuenta el resultado del proceso de investigación científica. No obstante, las autoridades ambientales y los particulares deben dar aplicación al principio de precaución conforme al cual, cuando exista peligro de daño grave e irreversible, la falta de certeza científica absoluta no deberá utilizarse como razón para postergar la adopción de medidas eficaces para impedir la degradación del medio 
ambiente. Este principio se encuentra en el numeral 6 del artículo 1 de la Ley 99 de 1993 y fue declarado exequible por la Corte Constitucional a través de la sentencia C-293 de 2002.

La tarea está en manos de las autoridades ambientales del país, en cabeza del ministerio del Medio Ambiente Vivienda y Desarrollo Territorial el cual dentro de sus funciones, establecidas en el artículo 5 de la Ley 99 de 1993 en los numerales 2, 9, 10, 11, 15, 17, 25, 26 y el parágrafo 1 en los que se incluyen aspectos relacionados con la formulación de la política nacional, la regulación de las condiciones generales para el saneamiento del medio ambiente a fin de impedir, reprimir, eliminar o mitigar el impacto de actividades contaminantes, deteriorantes o destructivas del entorno o del patrimonio natural, a las que deberán sujetarse los centros urbanos y las actividades industriales; así como la definición y regulación de los instrumentos y mecanismos necesarios para la prevención y control de los factores de deterioro ambiental, la evaluación de estudios ambientales, contratar la elaboración de estudios de investigación y de seguimiento de procesos biológicos y ambientales; al igual que establecer límites máximos permisibles de emisión, descarga, transporte o depósito de sustancias contaminantes y expedir las regulaciones ambientales para la distribución y el uso de sustancias químicas o biológicas utilizadas en actividades agropecuarias. De igual manera en el artículo 31 de esta ley se establecen las funciones de las CAR dentro de las que se incluye la de ejercer función de evaluación, control y seguimiento ambiental de los usos del agua y en materia de RESPEL las obligaciones establecidas en el Capítulo V del Decreto 4741 de 2005, en este sentido es importante que se tenga presente, que de una gestión inadecuada de los RESPEL en la cual se afecta la salud humana y el ambiente, dan lugar a la imposición de las medidas preventivas y sancionatorias consagradas en el artículo 85 de la Ley 99 de 1993, sin perjuicio de las acciones y civiles a que haya lugar.

\section{Discusión}

Los productos farmacéuticos humanos y de uso veterinario son contaminantes omnipresentes de las fuentes hídricas, con potenciales efectos perjudiciales sutiles en los organismos acuáticos, y posiblemente también en la salud humana. Los riesgos de los productos farmacéuticos, o compuestos farmacéuticamente activos, siguen siendo poco conocidos. Actualmente las acciones que la mayoría de países del mundo preocupados por esta amenaza, presentan un enfoque de principio de precaución adecuado para el control y vigilancia de los nuevos compuestos antes de su liberación al mercado y desde allí al medio receptor (Tijan et al, 2013), esto sumado a programas postconsumo de recolección de medicamentos, representan las combinaciones de estrategias de gestión actuales probablemente más eficaces en la mitigación de los riesgos presentados por los productos farmacéuticos.
El creciente interés y los esfuerzos realizados principalmente por los países desarrollados y sus organizaciones gubernamentales son evidencia de la amenaza que estos micronutrientes representan para sus poblaciones y el bienestar de los ecosistemas. Inicialmente se requiere definir el tipo de sustancias prioritarias a ser monitoreadas, dado que por razones técnicas y económicas, poder analizar, detectar y cuantificar la totalidad de sustancias presentes en el ambiente acuático sería, por decirlo de alguna manera, imposible; así mismo, habría una enorme dificultad para desarrollar programas de evaluación, control químico y restricción de estas sustancias, incluyendo metabolitos, subproductos y otros contaminantes de interés prioritario (Wernersson et al, 2015), ya que se requeriría un amplio espectro de criterios evaluativos, los cuales se desarrollan generalmente sustancia por sustancia, ignorando generalmente las consecuencias de la exposición simultánea a múltiples productos y subproductos químicos bioacumulables.

Por otro lado, aunque cada país y sociedad percibe y gestiona los riesgos de acuerdo con sus propios valores y prioridades (Rahman et al, 2009b), se espera que todas las partes interesadas en la investigación de los riesgos ambientales de los productos farmacéuticos tengan las mismas expectativas: esquemas para evaluar sustancias claros, transparentes, científicamente sólidos y comparables (si no idénticos), con la confianza y la disminución de la incertidumbre, en un nivel local, nacional e internacional (Straub et al 2012) y que a su vez brinden apoyo científico y técnico a las naciones en desarrollo, de tal manera que exista un control global sobre estos residuos.

\section{Referencias Bibliográficas}

Al-Odaini, N; Zakaria, M; Yaziz, M; Surif, S. (2010). Multi-residue analytical method for human pharmaceuticals and synthetic hormones in river water and sewage effluents by solid-phase extraction and liquid chromatography-tandem mass spectrometry. Journal of Chromatography A, 1217, p. 6791-6806.

Bellan, N; Pinto, T; Kaneko, T; Moretto, L; Santos, N. (2012). Critical analysis of the regulations regarding the disposal of medication waste. Brazilian Journal of Pharmaceutical Sciences. 48 . p. $507-$ 513.

Bila, D; Dezzoti, M. (2003). Fármacos no meio ambiente. Química Nova, 26(4), p. 523-530.

Bu, Q; Wang, B; Huang, J; Deng, S; Yu, G. (2013). Pharmaceuticals and personal care products in the aquatic environment in China: A review, Journal of Hazardous Materials. 262, p. 189-211.

Cai, K; Flliott, C; Phillips, D; Scippo, M; Muller, M; Connolly, L. (2012). Treatment of estrogens and androgens in dairy wastewater by a constructed wetland system. Water research 46, p. $2333-2343$.

Dai, G; Wang, B; Huang, J; Dong, R; Deng, S; Yu, G. (2015). Occurrence and source apportionment of pharmaceuticals and personal care products in the Beiyun River of Beijing, China, Chemosphere, 119, p. 1033-1039.

FAO \& PNUMA. (2015). Medidas de control del comercio internacional en virtud de los convenios de Basilea, Rotterdam y Estocolmo Secretariat of the Basel, Rotterdam and Stockholm Conventions, p.8.

Fisher, P \& Scott, R. (2008). Evaluating and controlling pharmaceutical emissions from dairy farms: a critical first step in developing a preventative management approach. Journal of Cleaner Production 16, p. 1437-1446.

Gemiliano, P. (2006). Análise comparativa de legislaçöes relativas à Qualidade da Água para consumo Humano na América do Sul [dissertação]. [Belo Horizonte (MG)]: Programa de Pós Graduação em Saneamento, Meio ambiente e Recursos Hídricos. Universidade Federal de Minas Gerais, p. 212. 
INS. Instituto Nacional de Salud. (2014). Vigilancia de la calidad del agua para consumo Humano Análisis comparativo Brasil y Colombia. Bogotá, D.C., Colombia. 20p.

Jelic', A; Petrovic', M. y Barcelo, D. (2012). Pharmaceuticals in Drinking Water. Pharmaceuticals in Drinking Water. 20:47-70.

Kampa, E; Dworak, T; Laaser, C y Vidaurre, R. (2010). European Regulations. Chapter 17. Green and Sustainable Pharmacy. K. Kümmerer, M. Hempel (eds.) p. $253-277$.

Kiran, A; Chiranjeevi, P; Mohanakrishna, G; Venkata, S. (2011). Natural attenuation of endocrine-disrupting estrogens in an ecologically engineered treatment system (EETS) designed with floating, submerged and emergent macrophytes. Ecological Engineering 37, p. $1555-1562$.

Kleywegt, S; Pileggi, V; Yang, P; Hao, C; Zhao, X; Thach, S; Cheung, P \& Whitehead, B. (2011). Pharmaceuticals, hormones and bisphenol A in untreated source and finished drinking water in Ontario, Canada - Occurrence and treatment efficiency. Science of the Total Environment 409, p. 1481-1488.

Lacina, P; Mravcov', L; V'avrov', M. (2013). Application of comprehensive two-dimensional gas chromatography with mass spectrometric detection for the analysis of selected drug residues in wastewater and surface water. Journal of Environmental Sciences, 25(1), p. 204-212.

Lee, L; Carmosini, N; Sassman, S; Dion, H y Sepúlveda, M. (2009). Agricultural Contributions of Antimicrobials and Hormones on Soil and Water Quality. Review. Advances in Agronomy, 93, p. 168.

Liu, S; Ying, G; Zhang, R; Zhou, L; Lai, H; Chen, Z. (2012). Fate and occurrence of steroids in swine and dairy cattle farms with different farming scales and wastes disposal systems. Environmental Pollution. 170, p. 190-201.

Manickum, T \& John, W. (2014). Occurrence, fate and environmental risk assessment of endocrine disrupting compounds at the wastewater treatment works in Pietermaritzburg (South Africa). Science of The Total Environment, 468-469(0), 584-597.

MAVDT. (2009). Ministerio de Ambiente, Vivienda y Desarrollo Territorial. Resolución 0371 (26, febrero, 2009). Por la cual se establecen los elementos que deben ser considerados en los Planes de Gestión de Devolución de Productos Posconsumo de Fármacos o Medicamentos Vencidos. Bogotá D.C.: El Ministerio $2009.9 \mathrm{p}$.

MAVDT. (2007). Ministerio de Protección Social. Decreto 1575 de 2007. Por el cual se establece el Sistema para la Protección y Control de la Calidad del Agua para Consumo Humano. Bogotá D.C.: El Ministerio 2007.15 p.

MAVDT. (2007). Ministerio de Ambiente, Vivienda y Desarrollo Territorial. Resolución 2115 de 2007. Por medio de la cual se señalan características, instrumentos básicos y frecuencias del sistema de control y vigilancia para la calidad del agua para consumo humano. Bogotá D.C.: El Ministerio 2007.23 p.

MAVDT. (2005). Ministerio de Ambiente, Vivienda y Desarrollo Territorial. Decreto 4741 de 2005 . 'Por el cual se reglamenta parcialmente la prevención y manejó de los residuos o desechos peligrosos generados en el marco de la gestión integral. Bogotá D.C.: El Ministerio 2005. 25 p.

MAVDT. (2005). Política Ambiental para la Gestión Integral de Residuos o Desechos Peligrosos. Ministerio de Ambiente, Vivienda y Desarrollo Territorial. República de Colombia. Pp 122

MAVDT. (1993). Congreso de la Republica. Ley 99 de 1993. Por la cual se crea el Ministerio del Medio Ambiente, se reordena el Sector Público encargado de la gestión y conservación del medio ambiente y los recursos naturales renovables, se organiza el Sistema Nacional Ambiental, SINA, y se dictan otras disposiciones. $59 \mathrm{p}$.

Marsalek, J. (2008). Pharmaceuticals and Personal Care Products (PPCP) in Canadian Urban Waters: A Management Perspective. En: P. Hlavinek et al. (eds.), Dangerous Pollutants (Xenobiotics) in Urban Water Cycle, p. 117-130.

McVey, E. (2012). Regulation of Pharmaceuticals in the Environment: The USA. Human Pharmaceuticals in the Environment: 49 Current and Future Perspectives, Emerging Topics in Ecotoxicology 4.p. 49-61.

Norman. (2012). Network of reference laboratories for monitoring of emerging environmental pollutants-Norman. http://www.norman-networknet

PNUMA. (2009). Convenio de Estocolmo. Sobre Contaminantes Orgánicos Persistentes. Secretariat of the Stockholmo Convention. p.64.

PNUMA. (2014). Convenio de Basilea sobre el Control de los Movimientos Transfronterizos de los Desechos Peligrosos y su eliminación. Secretariat of the Basel Convention International Environment House, p. 126.
Quevauviller, P; Carere, M; Polesello, S. (2012). Chemical monitoring activity for the implementation of the Water Framework Directive. Trends Anal Chem. 36, p. 1-184.

Rahman, M; Yanful, E; Jasim, S. (2009a). Endocrine disrupting compounds (EDCs) and pharmaceuticals and personal care products (PPCPs) in the aquatic environment: implications for the drinking water industry and global environmental health. Journal of Water and Health. 07 (2), p. $224-243$.

Rahman, M; Yanful, E; Jasim, S. (2009b). Occurrences of endocrine disrupting compounds and pharmaceuticals in the aquatic environment and their removal from drinking water: Challenges in the context of the developing world. Desalination 248, p. $578-585$.

Sharma, B; Bharat, G; Tayal, S; Nizzetto, L; Larssen, T. (2014a). The legal framework to manage chemical pollution in India and the lesson from the Persistent Organic Pollutants (POPs), Science of The Total Environment, 490 , p. $733-747$.

Sharma, B; Bharat, G; Tayal, S; Nizzetto, L; Larssen, T; Cupr, P. (2014b). Environment and human exposure to persistent organic pollutants (POPs) in India: A systematic review of recent and historical data, Environment International, 66, p. 48-64

Silva, L; Lino, C; Meisel, L. \& Pena, A. (2012). Ecopharmacovigilance. Pharmaceuticals in Drinking Water: 213-242.

Silva, C; Otero, M. \& Esteves, V. (2012). Processes for the elimination of estrogenic steroid hormone from water: A review. Environmental Pollution, 165, p. 38-58.

Straub, J. y Hutchinson, T. (2012). Environmental Risk Assessment for Human Pharmaceuticals: The Current State of International Regulations. Human Pharmaceuticals in the Environment: 17 Current and Future Perspectives, Emerging Topics in Ecotoxicology 4, p. $17-47$.

Tijani, J; Fatoba, O. \& Petrik, L. (2013). A Review of Pharmaceuticals and Endocrine-Disrupting Compounds: Sources, Effects, Removal, and Detections. Water, Air, \& Soil Pollution, 224 (11), p. 1-29.

Unión Europea. (2001). Decisión No 2455/2001/CEDEL PARLAMENTO EUROPEO Y DEL CONSEJO de 20 de noviembre de 2001 por la que se aprueba la lista de sustancias prioritarias en el ámbito de la política de aguas. Diario Oficial de las Comunidades Europeas. L. 331/1: $1-5$.

Unión Europea. (2004). Directive 2004/27/EC OF THE EUROPEAN PARLIAMENT AND OF THE COUNCIL of 31 March 2004 amending Directive 2001/83/EC on the Community code relating to medicinal products for human use. Official Journal of the European Union. L 136: $34-57$.

Unión Europea. (2013). Directiva 2013/39/UE del Parlamento Europeo y del Consejo, de 12 de agosto de 2013, por la que se modifican las Directivas 2000/60/CE y 2008/105/CE en cuanto a las sustancias prioritarias en el ámbito de la política de aguas. Diario Oficial de las Comunidades Europeas. 2013; L 226: p. 1-17.

USEPA. (2007). Method 1694: Pharmaceuticals and Personal Care Products in Water, Soil, Sediment, and Biosolids by HPLC/MS/MS. U.S. Environmental Protection Agency Office of Water Office of Science and Technology Engineering and Analysis Division (4303T) 1200 Pennsylvania, NW Washington, DC. p. 72.

USEPA. (2014a). Final 2012 and Preliminary 2014 Effluent Guidelines Program Plans. U.S. Environmental Protection Agency Office of Water Office of Science and Technology Engineering and Analysis Division (4303T) 1200 Pennsylvania, NW Washington, DC. p. 53.

USEPA. (2014b). Announcement of Preliminary Regulatory Determination for Contaminants on the Third Drinking Water Contaminant Candidate List. Federal Register. Vol. 79, No. 202, p. 62716

USEPA. (2015). Summary of Nominations for the Fourth Contaminant Candidate List. EPA 815-R-15-001. January, 2015.

Wennmalm, $\AA$ \& Gunnarsson, B. (2010). Chapter 16. Experiences with the Swedish Environmental Classification Scheme. Green and Sustainable Pharmacy. K. Kümmerer, M. Hempel (eds.) p. $243-$ 249.

Wernersson, A; Carere, M; Maggi, C. Tusil, P; Soldan, P; James, A, ... Kase, R. (2015). The European technical report on aquatic effectbased monitoring tools under the water framework directive. Environmental Sciences Europe, 27(1), p. 1-11.

Verlicchi, P; Al Aukidy, M; Zambello, E. (2012). Occurrence of pharmaceutical compounds in urban wastewater: Removal, mass load and environmental risk after a secondary treatment-A review, Science of The Total Environment, 429, p. 123-155.

WHO. World Health Organization. (2012). Pharmaceuticals in drinkingwater. WHO. Geneva, Switzerland, p. 52.

Zhang, D; Tan, S; Gersberg, R; Jern, W; keat, S. (2014). Removal of pharmaceuticals and personal care products in aquatic plantbased systems: A review. Environmental Pollution 184, p. 620639. 